\title{
Treatment of erectile dysfunction with sildenafil citrate (Viagra) in parkinsonism due to Parkinson's disease or multiple system atrophy with observations on orthostatic hypotension
}

\author{
I F Hussain, C M Brady, M J Swinn, C J Mathias, C J Fowler
}

\begin{abstract}
Objectives-To assess the efficacy and safety of sildenafil citrate (Viagra) in men with erectile dysfunction and parkinsonism due either to Parkinson's disease or multiple system atrophy.

Methods-Twenty four patients with erectile disease were recruited, 12 with Parkinson's disease and 12 with multiple system atrophy, into a randomised, double blind, placebo controlled, crossover study of sildenafil citrate. The starting dose was $50 \mathrm{mg}$ active or placebo medication with the opportunity for dose adjustment depending on efficacy and tolerability. The international index of erectile function questionnaire (IIEF) was used to assess treatment efficacy and a quality of life questionnaire to assess the effect of treatment on sex life and whole life. Criteria for entry included a definite neurological diagnosis and a standing systolic blood pressure of $90-180 \mathrm{~mm} \mathrm{Hg}$ and diastolic blood pressure of $50-110 \mathrm{~mm} \mathrm{Hg}$, on treatment if necessary. Blood pressure was taken at randomisation (visit 2) and crossover (visit 5) lying, sitting, and standing, before and 1 hour after taking the study medication in hospital.
\end{abstract}

Results-Sidenafil citrate was efficacious in men with parkinsonism with a significant improvement, as demonstrated in questionnaire responses, in ability to achieve and maintain an erection and improvement in quality of sex life. In Parkinson's disease there was minimal change in blood pressure between active and placebo medication. In multiple system atrophy, six patients were studied before recruitment was stopped because three men showed a severe drop in blood pressure 1 hour after taking the active medication. Two were already known to have orthostatic hypotension and were receiving treatment with ephedrine and midodrine but the third had asymptomatic hypotension. However, the blood pressures in all three had been within the inclusion criterion for the study protocol. Despite a significant postural fall in blood pressure after sildenafil, all patients with multiple system atrophy reported a good erectile response and were reluctant to discontinue the medication.

Conclusions-Sidenafil citrate $(50 \mathrm{mg})$ is efficacious in the treatment of erectile dysfunction in parkinsonism due to Parkinson's disease or multiple system atrophy; however, it may unmask or exacerbate hypotension in multiple system atrophy. As Parkinson's disease may be diagnostically difficult to distinguish from multiple system atrophy, especially in the early stages, we recommend measurement of lying and standing blood pressure before prescribing sildenafil to men with parkinsonism. Furthermore, such patients should be made aware of seeking medical advice if they develop symptoms on treatment suggestive of orthostatic hypotension.

(f Neurol Neurosurg Psychiatry 2001;71:371-374)

Keywords: erectile dysfunction; Parkinson's disease; multiple system atrophy; viagra; orthostatic hypotension

Estimates of the prevalence of erectile dysfunction in men with Parkinson's disease show that it is a significant problem, affecting $60 \%$ of a group of men compared with an age matched healthy non-parkinsonian group, in whom the prevalence was $37.5 \% .^{1}$ Typically the problem only affects men some years after the neurological disease has been established. ${ }^{2}$ A survey of young patients with Parkinson's disease (mean age 49.6 years) that included their partners also, showed a high level of sexual dysfunction with the most severely affected couples being those in which the patient was male. Erectile dysfunction and premature ejaculation were complaints in a significant proportion although in general terms sexual dysfunction seemed to be multifactorial with no single cause identified. ${ }^{3}$ There is experimental evidence that dopaminergic mechanisms are involved in determining libido and causing penile erection ${ }^{4}$ and the possible role of a central deficiency of dopamine in this disease remains to be examined.

In many cases of multiple system atrophy, urinary incontinence and other autonomic disturbances may precede the onset of major neurological symptoms but urogenital symptoms are usually manifest before symptoms of orthostatic hypotension. ${ }^{5}$ In a study of the clinical features and treatment of genitourinary dysfunction in 62 patients with multiple system atrophy, $96 \%$ of the men had erectile dysfunction, with this as the first symptom in $37 \% .{ }^{6}$ In another study of 100 patients with multiple 
system atrophy, autonomic symptoms, including erectile dysfunction, were the initial features of the disease in $41 \%$ and had subsequently developed in $97 \%$ of male patients; symptomatic orthostatic hypotension, although present in $68 \%$, was severe and caused fainting in only $15 \%$ of patients. ${ }^{7}$ The symptoms caused by orthostatic hypotension have been studied in 40 patients with multiple system atrophy, and despite an orthostatic blood pressure fall of $>30 \mathrm{~mm} \mathrm{Hg}$ less than $50 \%$ have syncope (unlike pure autonomic failure), whereas others only have symptoms such as fatigue, lethargy, and weakness. ${ }^{8}$

The extensive clinical database that already exists for sildenafil citrate demonstrates that it is efficacious in the management of erectile dysfunction, and safe when appropriately used. The contraindication with any form of nitrate therapy is absolute because a synergistic response can result in severe hypotension. ${ }^{9}$ In normal volunteer studies sildenafil reduced mean maximum supine systolic and diastolic blood pressure by about $8 \mathrm{~mm} \mathrm{Hg}$ and $5 \mathrm{~mm}$ $\mathrm{Hg}$ without an effect on the heart rate.

The safety and efficacy of sildenafil for the treatment of erectile dysfunction in parkinsonism due to Parkinson's disease and multiple system atrophy had not been studied before. Although multiple system atrophy is less common than Parkinson's disease, it is a disease in which most men have erectile dysfunction and because orthostatic hypotension may be a complication, such a study is of particular relevance.

\section{Methods}

PATIENTS

Twenty four male patients with a well documented history of erectile dysfunction, 12 with a diagnosis of Parkinson's disease, and 12 with multiple system atrophy were recruited into the study; informed consent was obtained before the patient was screened. The protocol was approved by the joint ethics committee at the National Hospital for Neurology and Neurosurgery and the Institute of Neurology. A diagnosis of Parkinson's disease or multiple system atrophy had previously been made by a consultant neurologist after investigation and according to accepted inclusion criteria.

Patients were excluded if they had no stable sexual partner, penile deformity, other sexual or psychological disorders, or a known history of alcohol or drug dependence. Patients with diabetes, retinitis pigmentosa, a history of stroke or myocardial infarction, or significant cardiac history were also excluded. Treated resting and standing systolic blood pressure was required to be between 90 and $180 \mathrm{~mm} \mathrm{Hg}$ and diastolic BP between 50 and $110 \mathrm{~mm} \mathrm{Hg}$.

Written documentation from the patient's family doctor was required to ensure that they were not on any nitrate medication, and lipid abnormality and thyroid, renal, hepatic, and haematological disease were excluded by screening blood tests.

The median age of the men with Parkinson's disease was 61 years (range 48-68) and for the men with multiple system atrophy, 54 years (range 46-61). The median duration of erectile dysfunction in the men with Parkinson's disease was 54 months (range 12-72) and 57 months (range 24-90) in the men with multiple system atrophy.

\section{QUESTIONNAIRES}

Efficacy was judged by the international index of erectile function (IIEF), a psychometrically validated 15 item self administered questionnaire covering the domains of erection, orgasm, desire, intercourse satisfaction, and overall satisfaction with sex life. ${ }^{10}$ This includes the ability to obtain an erection (question 3) and the ability to maintain an erection adequate for intercourse on a scale of $0-5$ (question 4), where "almost always" or "always" scores 5. In normal healthy men the mean responses for these questions are 4.0 and 4.2 .

A quality of life (QoL) questionnaire was also used. This assessed eight factors including whole life, vocational and financial situation, and sex life. Responses range from 0 to 5 (very dissatisfying to very satisfying).

The patient's partner was also given a brief questionnaire to complete independently.

The primary efficacy end points were the responses to questions 3 and 4 of the IIEF and responses to the QoL questionnaire.

\section{TRIAL DESIGN}

The trial was designed as a randomised, double blind, placebo controlled, crossover study with flexible dosage starting at $50 \mathrm{mg}$ active medication or placebo and was conducted over a 24 week period in each patient. The dose could be titrated up to $100 \mathrm{mg}$ or down to 25 mg depending on efficacy and tolerability. Medication was on an "as needed" basis 1 hour before sexual activity, and patients were provided with three tablets a week but advised not to take more than one each day.

At visit 1, consent was obtained before demographic data and screening blood samples were taken. Patients were advised to stop all therapy for erectile dysfunction and sexual activity was encouraged.

After a 4 week run in period (visit 2), patients filled in a baseline IIEF questionnaire and were randomised to receive either $50 \mathrm{mg}$ sildenafil citrate or placebo medication. The first dose was taken in the department and the heart rate with lying, sitting and standing blood pressures were recorded before and 1 hour after dosing. At visits 3 and 4 the dose was titrated up or down if necessary, depending on efficacy and tolerability. At visit 5, after 10 weeks of treatment, patients attended for the crossover and the same procedure was repeated, visits 6 and 7 being similar to visits 3 and 4 . At the final visit, the patients repeated the IIEF questionnaire and had a further blood test.

Medication was kept in the pharmacy together with the randomisation detail and only enough was dispensed until the next visit. Patients were required to keep a dosing log.

Statistical analysis was carried out by Pfizer, New York, USA. A crossover analysis of variance (ANOVA) model was utilised with terms for sequence, patient, period, and 

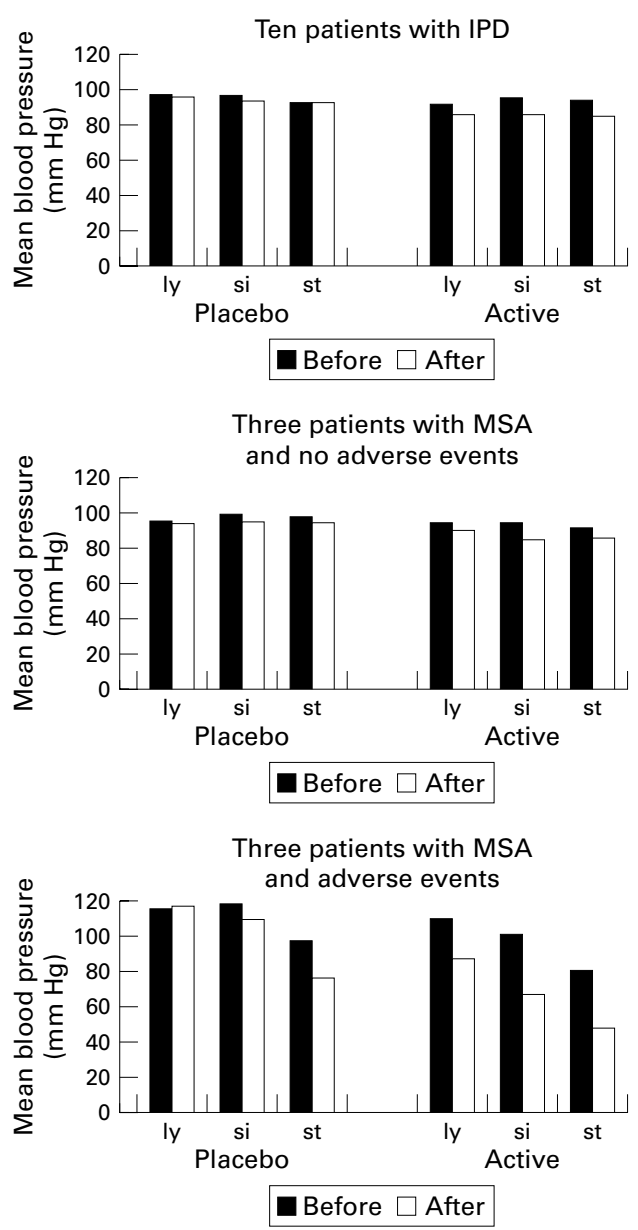

Figure 1 Average mean (diastolic+one third difference in systolic and diastolic pressure) lying (ly), sitting (si), and standing (st) blood pressures in 10 patients with

Parkinson's disease who tolerated the medication with minimal effect on the blood pressures (top panel); three patients with multiple system atrophy who tolerated the medication without an adverse effect on their blood pressures (middle panel); and three patients who developed a symptomatic fall in their blood pressures 1 hour after treatment with Sildenafil (bottom panel).

treatment. The analysis was of a change from baseline in efficacy variables.

Mean blood pressure, the variable shown in figure 1 , was calculated as the diastolic pressure plus one third of the difference between systolic and diastolic pressures.

\section{Results}

PARKINSON'S DISEASE

Of the 12 men with Parkinson's disease recruited, 10 completed the study, one withdrew consent at visit 3 and another was found to have carcinoma of the lung during the run in period and was not treated. Four of 10 patients were randomised to receive the placebo for the first 10 weeks and the remainder for the second 10 weeks. Nine of 10 patients reported a good response to sildenafil citrate, eight titrated up to $100 \mathrm{mg}$ and one titrated down to $25 \mathrm{mg}$. One patient reported lack of efficacy and returned to intracavernosal injections at the conclusion of the study.

Overall the baseline and 10 week postplacebo data for IIEF (questions 3 and 4) and the QoL for whole life and sex life were similar.
Table 1 The responses to $Q 3$ and $Q 4$ of the IIEF questionnaire and the quality of whole life and sex life for placebo medication and sildenafil citrate in men with parkinsonism.

\begin{tabular}{|c|c|c|c|}
\hline & $\begin{array}{l}\text { Placebo } \\
(n=16)\end{array}$ & $\begin{array}{l}\text { Sildenafil } \\
(n=14)\end{array}$ & $\begin{array}{l}\text { Crossover } \\
\text { ANOVA model }\end{array}$ \\
\hline \multicolumn{4}{|l|}{ IIEF: } \\
\hline Question 3 & 1.56 & 3.71 & $\mathrm{p}=0.0095$ \\
\hline Question 4 & 1.44 & 3.79 & $\mathrm{p}=0.0041$ \\
\hline \multicolumn{4}{|l|}{ Quality of life: } \\
\hline Whole life & 4.69 & 4.93 & $\mathrm{p}=0.3505$ \\
\hline Sex life & 2.19 & 4.21 & $\mathrm{p}=0.0073$ \\
\hline
\end{tabular}

There was a significant improvement over placebo for both Q3 (obtaining an erection) and Q4 (maintaining an erection). Although sildenafil did not affect the quality of whole life there was a significant improvement in the quality of sex life. The $p$ values are based on a crossover ANOVA model.

The same responses after 10 weeks of active medication starting with $50 \mathrm{mg}$ and titrated up to $100 \mathrm{mg}$ in most patients showed a marked and significant improvement in being able to obtain (question 3) and maintain an erection (question 4) for sexual intercourse. Although there was little change in the whole life QoL there was a marked improvement in sex life (table 1). The partner questionnaire response confirmed the improvement. There were no serious adverse events over the study period. One patient reported headache and a warm feeling after taking the active medication but did not discontinue it.

The predose and postdose measurements of blood pressure for the placebo medication showed no postural drop in mean blood pressure (fig 1). One hour after active medication there was a change in the average mean blood pressures of $5 \mathrm{~mm} \mathrm{Hg}, 9 \mathrm{~mm} \mathrm{Hg}$, and 9 $\mathrm{mm} \mathrm{Hg}$ in the lying, sitting, and standing positions respectively.

\section{MULTIPLE SYSTEM ATROPHY}

In the multiple system atrophy group, six patients were studied before recruitment was stopped. Four of six were randomised to receive the placebo for the first 10 weeks and active medication for the second 10 weeks. Baseline scores for IIEF questions 3 and 4 were low, with marginal improvement after placebo but they improved significantly after active medication, as did the quality of sex life (table 1).

Three patients had a severe fall in blood pressure 1 hour after sildenafil (one at visit 2 and two at visit 5), the standing blood pressure falling from $128 / 85$ to $65 / 55,104 / 60$ to $56 / 32$, and $115 / 70$ to $55 / 39$ respectively. All three remained conscious but had severe symptoms and signs of orthostatic hypotension, feeling unwell and unable to stand for more than a few seconds. Each patient had to wait (for 4 hours in one patient) until the drop in blood pressure became tolerable. Figure 1 shows the average mean blood pressure changes in this group. On the basis of this adverse event a decision was taken to unblind the study and discontinue recruitment.

\section{Discussion}

We have shown that sildenafil citrate is efficacious in the treatment of erectile dysfunction in patients with parkinsonism having Parkinson's disease or multiple system atrophy. 
Sildenafil enhanced erectile function, enabling intercourse, which resulted in an improved quality of sex life. It was well tolerated in Parkinson's disease and the minor adverse effects of headache and flushing were transient and required no treatment. However, in multiple system atrophy, sildenafil caused a severe fall in blood pressure in half of the treated patients, resulting in early termination of the study. The adverse event was recognised because the first dose of all medication was taken under supervision in the department. Two of these patients were already receiving treatment with ephedrine and midodrine for orthostatic hypotension and all three patients with this adverse event had a significant fall in postural blood pressure before sildenafil, which potentiated the response. However, on treatment their lying, sitting, and standing blood pressures were within the inclusion criteria for the study protocol. Despite severe and symptomatic hypotension, all three wanted to continue to use the medication and were dismissive of the suggestion that sildenafil was unsafe for them. One patient obtained it via the internet although he was made aware of the possible hazards. The three men with multiple system atrophy who tolerated the medication well had minimal postural fall in blood pressure and although the lying, sitting, and standing pressures were lower with active medication there was no postural accentuation (fig 1). Thus, the presence of orthostatic hypotension and its magnitude was predictive of a further fall and adverse reaction to sildenafil.

Debate continues about the mechanisms causing erectile dysfunction in multiple system atrophy, and a mechanism which includes impairment of dopaminergic mediated pathways in the CNS - which are being increasingly recognised as important for erectile functionshould be considered. The demonstrated efficacy of sildenafil in the presence of orthostatic hypotension indicates that hypotension itself, with reduced filling pressures, cannot be causal.
There are often difficulties in early diagnosis and separation of Parkinson's disease from multiple system atrophy. Although orthostatic hypotension is uncommon in early Parkinson's disease, this is not the case in multiple system atrophy. We recommend measurement of lying and standing blood pressure before prescribing sildenafil to men with parkinsonism. Furthermore, such men should be followed up with blood pressure measurements and made aware of the need to seek medical advice if they develop symptoms suggesting orthostatic hypotension.

This study was made possible by an investigator grant from Pfizer. We also thank Pfizer for carrying out the statistical analyses, Mrs K Bleasdale-Barr, Autonomie Unit, and Mrs E M Boal, Uro-Neurology, National Hospital for Neurology and Neurosurgery, for assistance in the assessment and treatment of patients in this study.

1 Singer C, Weiner WJ, Sanchez-Ramos JR, et al. Sexual dysfunction in men with Parkinson's disease. Fournal of Neurological Rehabilitation 1989;3:199-204.

2 Chandiramani VA, Palace J, Fowler CJ. How to recognise patients with parkinsonism who should not have urological surgery. British fournal of Urology 1997;80:100-4.

3 Brown RG, Jahanshahi M, Quinn N, et al. Sexual function in patients with Parkinson's disease and their partners. $7 \mathrm{Neu}$ rol Neurosurg Psychiatry 1990;53:480-6.

4 Heaton J. Central neuropharmacological agents and mechanisms in erectile dysfunction: the role of dopamine. Neurosci Behav Rev 2000;24:561-9.

5 Sakakibara R, Hattori T, Uchiyama $\mathrm{T}$, et al. Urinary dysfunction and orthostatic hypotension in multiple system atrophy: which is the more common and earlier manifestation? f Neurol Neurosurg Psychiatry 2000;68:65-9.

6 Beck RO, Betts CD, Fowler CJ. Genito-urinary dysfunction in multiple system atrophy: clinical features and treatment in 62 cases. F Urol 1994;151:1336-41.

7 Wenning G, Shlomo Y, Magalhaes M, et al. Clinical features and natural history of multiple system atrophy. Brain 1994; 117:835-45.

8 Mathias CJ. Cardiovascular autonomic dysfunction in parkinsonian patients Clin Neurosci 1998;5:153-66.

9 Cheitlin MD, Hutter AM Jr, Brindis RG, et al. ACC/AHA expert consensus document. Use of sildenafil (viagra) in patients with cardiovascular disease. /American Heart Association. F Am Coll Cardiol 1999;33:273-82.

10 Rosen R C, Riley A, Wagner G, et al. The international index of erectile function (IIEF): a multidimensional scale for assessment of erectile dysfunction. Urology 1997;49: $822-30$ 